\title{
Meta
}

Journal des traducteurs

Translators' Journal

\section{Scott L. Montgomery (2000): Science in Translation. Movements of Knowledge through Cultures and Times, Chicago and London, The University of Chicago Press, xii-326 p.}

\section{André Clas}

Volume 46, numéro 1, mars 2001

Traduction médicale et documentation / Medical translation and documentation

URI : https://id.erudit.org/iderudit/002267ar

DOI : https://doi.org/10.7202/002267ar

Aller au sommaire du numéro

Éditeur(s)

Les Presses de l'Université de Montréal

ISSN

0026-0452 (imprimé)

1492-1421 (numérique)

Découvrir la revue

Citer ce compte rendu

Clas, A. (2001). Compte rendu de [Scott L. Montgomery (2000): Science in Translation. Movements of Knowledge through Cultures and Times, Chicago and London, The University of Chicago Press, xii-326 p.] Meta, 46(1), 176-178.

https://doi.org/10.7202/002267ar d'utilisation que vous pouvez consulter en ligne. 


\section{Compte rendu}

Scott L. Montgomery (2000): Science in Translation. Movements of Knowledge through Cultures and Times, Chicago and London, The University of Chicago Press, xii-326 p.

Voilà un livre qui comble une grande lacune et qui rend justice - enfin, et il faut en féliciter l'auteur — au rôle fondamental joué par la traduction dans l'histoire du savoir scientifique à travers les âges - Antiquité, Moyen Âge et Époque moderne et qui montre l'importance de la traduction dans l'élaboration de la science occidentale. L'auteur a travaillé, à temps partiel, pendant plus de dix ans comme traducteur scientifique et technique, et son expérience, extrêmement diversifiée, lui a montré sans restriction que la traduction intervient dans tous les domaines du savoir et également dans la diffusion des connaissances scientifiques. Signalons que l'auteur, qui est géologue consultant, écrivain et chercheur scientifique indépendant, a publié plusieurs ouvrages, notamment Minds for the Making: The Role of Science in American Education, 1750-1990; The Scientific Voice et The Moon and the Western Imagination. Sa grande érudition lui a permis de nous présenter un livre qui comble un vide sur la connaissance du rôle de la traduction dans les sciences, dans leur développement et dans la diffusion des connaissances scientifiques et leurs conséquences linguistiques, sociales, politiques et économiques. C'est un ouvrage fascinant à lire, très bien documenté - plus de 400 références bibliographiques — avec un index détaillé, des citations attestées, des notes de bas de page apportant des précisions ou des compléments d'information, bref un modèle de travail de recherche et d'érudition. On ne peut que souhaiter que l'ouvrage devienne un manuel de lecture obligatoire pour tous les étudiants dans toutes les écoles de traduction. Il s'inscrit certes dans les cours d'histoire de la traduction et, par exemple, dans les séminaires de terminologie comme exemple d'évolution et de développement de la créativité linguistique.

Science in Translation est une étude de cas et utilise comme exemples la connaissance et le développement de l'astronomie de l'époque originelle grecque aux transformations du cosmos médiéval et les origines de la science dans le monde non occidental où le Japon sert de pays d'investigation. Le livre est divisé en trois grandes parties: les cieux à travers le temps et l'espace (The Heavens through Time and Space) avec quatre chapitres (The Era of Roman Translation; Astronomy in the East; The Formation of Arabic Science; Eighth through Tenth Centuries; Era of Transfer into Latin), la science dans le monde non occidental (Science in the Non-Western World) avec deux chapitres (Record of Recent Matters; Japanese Science in the Making) et le contexte contemporain (The Contemporary Context) avec deux chapitres (Issues and Examples for the Study of Scientific Translation Today et les Conclusions (gained in Translation)).

Aucun auteur classique ne nous est parvenu dans ses écrits originels et ce qui existe montre une "communauté textuelle» formée de traducteurs grecs, byzantins, arabes et européens, de scribes, d'éditeurs et de commentateurs qui sont tous impliqués dans l'interprétation et l'utilisation des écrits. Par exemple, les écrits de Ptolémée sur

Meta, XLVI, 1, 2001 
les mathématiques et l'astronomie ont été copiés, révisés et personnifiés. Sans nul doute, les progrès techniques ont également joué un rôle; et l'utilisation de matériaux peu durables rendait la reproduction obligatoire pour chaque génération et en faisait dès lors une œuvre contemporaine. La possibilité de recréer en latin les meilleurs écrits grecs donnait également l'occasion de forger de nouveaux modèles qui pouvaient remplacer les originaux. Pline le Jeune recommandait la traduction comme moyen de formation. Les sources originelles ne sont plus considérées comme sacrées, mais comme des œuvres qu'il fallait égaler et même surpasser. La traduction devient ainsi un procédé de substitution d'influence latine à celle des Grecs. Cicéron traduit Platon et cherche à produire la meilleure adaptation romaine utilisable par toute la res publica: utilisation de termes tirés d'autres domaines; utilisation de mots latins généraux; formation de mots composés; adaptations poétiques de mots latins ordinaires; emprunts phonétiques directs au grec. L'astronomie grecque est transformée en étude de cas et utilise comme exemples la connaissance et le développement de l'astronomie de l'époque originelle grecque aux transformations du cosmos médiéval et les origines de la science dans le monde non occidental, ce à quoi le Japon sert de pays d'investigation. La terminologie de l'astronomie, sans nul doute la science la plus ancienne avec plus de cinq millénaires d'existence dans la recherche de formes célestes et la possibilité de les prédire, permet de retracer l'histoire de la constitution de cette science. L'astronomie grecque est transformée par les Romains, grâce aux traducteurs, notamment Cicéron, mais également grâce aux compilateurs, aux auteurs de manuels. C'est la Naturalis Historia de Pline l'Ancien, cette œuvre monumentale de plus de 36 livres donnant la somme des connaissances de cette époque romaine - l'œuvre sera reprise, traduite et adaptée dans les diverses langues occidentales au XVI ${ }^{\mathrm{e}}$ siècle - , qui imprime ainsi son influence sur toute la pensée occidentale. Si les Romains cherchaient à simplifier le vocabulaire utilisé dans les textes des sources grecques, les traducteurs, au contraire, introduisaient le vocabulaire scientifique de leur époque.

Il ne faut cependant pas surestimer la part romaine dans la transmission des connaissances de l'astronomie; il faut tenir compte des apports grecs, plus particulièrement les érudits de Byzance et des communautés chrétiennes de Syrie qui parlaient le grec et le syriaque. À la transmission romaine des connaissances de l'astronomie, s'ajoute l'apport des traductions du grec en syriaque puis en arabe. Aux $\mathrm{v}^{\mathrm{e}}$ et $\mathrm{vI}^{\mathrm{e}}$ siècles, sans doute à cause des interférences de l'Église orthodoxe byzantine vis-à-vis des Nestoriens, des purges systématiques des empereurs Zénon et Justinien, les communautés se sont retirées vers l'est, vers les zones périphériques de l'Empire byzantin et en Perse, où elles ont établi des écoles et ont traduit les textes grecs. On sait, par exemple, que le syriaque a servi de langue source au $\mathrm{IX}^{\mathrm{e}}$ siècle pour des traductions en arabe lors du califat des Abbassides (705-1258). La traduction d'œuvres grecques en syriaque ne s'est point arrêtée avec la conquête arabe après 632. En fait, elle a augmenté, plus particulièrement au $\mathrm{IX}^{\mathrm{e}}$ siècle lorsque les califes des Abbassides ont encouragé de façon massive le transfert du savoir grec en arabe, le syriaque étant un intermédiaire. Il est vrai que la région du Moyen-Orient était riche linguistiquement: en plus de divers dialectes de l'araméen, comme le syriaque, il y avait encore l'hébreu, l'arabe, l'arménien et le géorgien pour ne nommer que les plus importantes langues. L'intérêt se portait spécifiquement vers la philosophie (Aristote), l'histoire et les sciences. L'arabe avait une raison religieuse particulière: les sciences islamiques, à 
savoir l'étude du Coran et ses applications à la littérature, au droit et à la politique, étaient séparées de l'enseignement grec. La traduction faisait du syriaque une langue riche et développée, une langue scientifique concurrente du grec. L'astronomie arabe s'enrichissait des apports des langues grecques et syriaques. La région sous domination arabe bénéficiait des apports des Syriens, des Grecs, des Perses, des Juifs, des Hindous et des Arméniens, tous héritiers de la science grecque, modifiée, adaptée aux diverses traditions et besoins particuliers par des échanges de connaissances entre les $\mathrm{II}^{\mathrm{e}}$ et $\mathrm{VI}^{\mathrm{e}}$ siècles. Lieu d'emprisonnement des soldats grecs de l'empereur romain Valérien, prisonniers syriens capturés lors de la prise d'Antioche, centre culturel de l'Empire romain oriental et lieu de refuge des réfugiés des purges de Zéno, Judishapur était un centre de traduction important pendant cinq siècles. Son annexion par les Arabes donnait une nouvelle dimension aux connaissances intellectuelles et aux transferts linguistiques en astronomie en apportant une source gréco-indienne, en pehlvi et en sanscrit, du savoir grec en arabe, le syriaque étant un intermédiaire. Un siècle plus tard, Bagdad devint, grâce à l'appui des califes abbassides, un centre de traduction florissant, un des lieux d'enseignement scientifique des plus importants. Les "sciences islamiques", c'est-à-dire la théologie, le droit, les langues, l'exégèse s'appuyant sur le Coran, étaient séparées des enseignements tirés du grec, dont on recherchait la naturalisation. C'est le «Livre» qui imposait une série d'intérêts intellectuels et qui allait se répandre dans diverses directions et dont le débat moderne porte encore des traces. Mais l'histoire n'est pas stationnaire et, peu à peu, on voit apparaître de nouveaux centres. La technologie se développe, les manuscrits se multiplient, l'urbanisation progresse, les universités naissent (Paris, Bologne), la fabrication du papier est introduite en Europe, les écoles se multiplient, le pèlerinage fait son apparition, bref les conditions de vie changent. Les croisades ont eu lieu. Les Mongols s'avancent vers l'ouest. On redécouvre le grec et le latin, et l'Europe intègre dans de nouvelles traductions les travaux des Arabes, des Juifs, des Grecs, des Romains ainsi que, de façon indirecte, le savoir des Indiens et des Perses. La Sicile et l'Italie du Sud sont devenues d'importants centres de traduction. Là encore, on montre que la traduction est le vecteur de la connaissance qui s'enrichit par son cheminement au cours du temps et à travers diverses langues et cultures et donne naissance à la science moderne.

La deuxième partie de l'ouvrage traite de l'époque contemporaine et montre les vicissitudes par lesquelles passe le japonais pour constituer un ensemble de facteurs permettant à la science moderne de se développer.

La conclusion de l'ouvrage peut se résumer par les mots de l'auteur lorsqu'il écrit: «Translation easily becomes a new eye cast upon the delights and difficulties of science scholarship. Through its lens, one discern that mobilities of knowledge are integral to the substance and crucial to any understanding of the scientific past and present.» (p. 294).

C'est à n'en pas douter, un livre d'une grande richesse, tant en exemples qu'en réflexions et qui illustre avec acuité le développement des connaissances à travers les langues et les époques. C'est un livre à lire et à relire. La matière est importante, elle est nôtre, elle est notre histoire. 